\title{
CONSTRUCTAL DESIGN AND SIMULATED ANNEALING EMPLOYED FOR GEOMETRIC OPTIMIZATION OF A Y- SHAPED CAVITY INTRUDED INTO CONDUCTIVE WALL
}

\author{
G. V. Gonzales ${ }^{\mathrm{a}}$, \\ E. D. dos Santos ${ }^{a}$, \\ L. R. Emmendorfer ${ }^{\text {a }}$, \\ L. A. Isoldi ${ }^{\text {a }}$, \\ E. S. D. Estrada ${ }^{b}$, \\ and L. A. O. Rochab \\ ${ }^{\mathrm{a}}$ Universidade Federal do Rio Grande \\ Programa de Pós-Graduação em Modelagem \\ Computacional (PPGMC) \\ Av. Itália, Km 8, Campus Carreiros \\ CEP 96021-900, Rio Grande, RS, Brasil \\ gillgonzales@ifsul.edu.br \\ ${ }^{b}$ Universidade Federal do Rio Grande do Sul \\ Departamento de Engenharia Mecânica \\ Rua Sarmento Leite, 425, Centro \\ CEP 90050-170, Porto Alegre, RS, Brasil \\ Received: March 04, 2015 \\ Revised: April 06, 2015 \\ Accepted: May 07, 2015
}

\section{ABSTRACT}

The problem study here is concerned with the geometrical evaluation of an isothermal Y-shaped cavity intruded into conducting solid wall with internal heat generation. The cavity acts as a sink of the heat generated into the solid. The main purpose here is to minimize the maximal excess of temperature $(\theta \max )$ in the solid. Constructal Design, which is based on the objective and constraints principle, is employed to evaluate the geometries of Y-shaped cavity. Meanwhile, Simulated Annealing (SA) algorithm is employed as optimization method to seek for the best shapes. To validate the SA methodology, the results obtained with SA are compared with those achieved with Genetic Algorithm (GA) and Exaustive Search (ES) in recent studies of literature. The comparison between the optimization methods (SA, GA and ES) showed that Simulated Annealing is highly effective in the search for the optimal shapes of the studied case.

Keywords: constructal theory, simulated annealing, geometric optimization, heat transfer

\section{NOMENCLATURE}

A area, $\mathrm{m}^{2}$

$\mathrm{H}$ height, $\mathrm{m}$

$\mathrm{k} \quad$ body thermal conductivity, $\mathrm{W} \mathrm{m}^{-1} \mathrm{~K}^{-1}$

L length, $\mathrm{m}$

$\mathrm{L}_{0} \quad$ elemental length, $\mathrm{m}$

$\mathrm{L}_{1} \quad$ stem length, $\mathrm{m}$

q heat current, $\mathrm{W}$

$\mathrm{t}$ thickness, $\mathrm{m}$

$t_{0} \quad$ elemental thickness, $m$

$\mathrm{t}_{1} \quad$ stem thickness, $\mathrm{m}$

$\mathrm{T}$ temperature, $\mathrm{K}$

W width, $m$

$\mathrm{x}, \mathrm{y}$ cartesian coordinates, $\mathrm{m}$

\section{Greek symbols}

$\alpha$

angle formed by the tributary branch of the Y-

shaped cavity and horizontal axis, rad

$\theta \quad$ dimensionless temperature, eq. (7)

$\phi \quad$ area fraction

$\psi \quad$ auxiliary area fraction

\section{Subscripts}

$\begin{array}{ll}\text { aux } & \text { auxiliary } \\ \mathrm{c} & \text { cavity } \\ \mathrm{m} & \text { once minimized } \\ \mathrm{mm} & \text { twice minimized } \\ \mathrm{mmm} & \text { three times minimized } \\ \mathrm{mmm} & \text { fourth times minimized } \\ \mathrm{o} & \text { optimal } \\ \text { oo } & \text { twice optimized } \\ \text { ooo } & \text { three times optimized } \\ \text { oooo } & \text { fourth times optimized } \\ 0 & \text { tributary branches } \\ 1 & \text { stem }\end{array}$

Superscripts

( ) dimensionless variables, eq. (8)

\section{INTRODUCTION}

With the technological advancement of the industry and the miniaturization of the systems and electronics circuits, new needs and constraints 
emerged in heat transfer problems. The traditional methods for cooling of electronic devices do not comply with the actual scales required for their working. According to Bejan (2003), the viable solution is the utilization of cooling ducts, cavities or high conductive pathways intruded in the medium with heat generation. The problem studied here is concerned with a solid wall with internal uniform heat generation which can be cooled solely by means of the isothermal Y-shaped cavity intruded into the wall. The present research employed Constructal Design method to guide the evaluation of geometrical shape of the cavity which minimizes the maximal excess of temperature $\left(\theta_{\max }\right)$ (Bejan, 2000; Bejan and Lorente, 2008). The search for the optimal geometries is performed with the combinatorial optimization algorithm Simulated Annealing (SA) (Kirkpatrick et al.,1983; Eglese, 1990).

Constructal Theory is the view that geometry patterns of flux systems in nature emerged by a physical principle, i.e., shape and structure generated in animate and inanimate systems are deterministic and not the result of random. Constructal Theory, proposed by Adrian Bejan (1996), states that geometry in any flux systems follows the Constructal Law, which states that "For a finite-size flow system to persist in time (to live), its configuration must evolve in such a way that provides easier access to the currents that flow through it". The form to employ Constructal Law for geometric evaluation of flux systems is the Constructal Design (Bejan, 2000), which is a method based on objectives and restrictions used to minimize the imperfections of the investigated systems. Recently, several C, T, Yshaped cavities, as well as, complex and multiple cavities has been studied by means of Constructal Design, see Refs. (Biserni et al.,2004; Xie et al.,2010a; Lorenzini et al., 2011; Estrada et al.,2012; Lorenzini et al.,2012; Lorenzini et al. 2013; 11. Hajmohammadi et al.,2013; Lorenzini et al., 2014a; Lorenzini et al., 2014b).

In the study of Biserni et al. (2004) the geometry of $\mathrm{C}$ and $\mathrm{T}$-shaped cavities intruded into a rectangular solid with internal heat generation was optimized. The T-shaped cavity has a higher performance than $\mathrm{C}$-shaped one, i. e., the most complex cavity with a higher penetration in the solid domain had the best efficiency. In the work of Xie et al. (2010) it was applied Constructal Design to geometric optimization of a T-shaped cavity intruded into a trapezoidal solid domain with uniform heat generation. As performed in the previous study of Biserni et al. (2004) the external surfaces of solid domain are considered adiabatic. In the work of Lorenzini et al. (2009) a complex T-Y-shaped cavity has been optimized and authors concluded that the complex cavity led to a thermal performance $108 \%$ superior than that reached with a C-shaped configuration, for the same conditions and dimensions of the problem. Furthermore, in the researches of Lorenzini et al. (2011), the geometric optimization of a Y-shaped cavity demonstrated that this geometry has a performance nearly $109 \%$ higher than that predicted with the elemental cavity.

The above mentioned studies allowed the observation that most complex geometries tend to conduct the flux system to the highest efficiencies. For the cavity problems, systems which minimizes the maximal excess of temperature. However, as more complex are the cavities more degrees of freedom they have and higher computational effort is required to solve the problem for all geometric possibilities and, consequently, find the optimal geometry. Recently studies (Lorenzini et al., 2014a; Lorenzini et al., 2014b), employed search algorithms to seek for the best shapes in alternative at conventional method of Exhaustive Search (ES), widely used in previous studies of cavities. In both studies it was applied Genetic Algorithm (GA) for the geometric optimization of Y-shaped cavity intruded into a solid with constant heat generation. In the work of Lorenzini et al. (2014a) performed a comparison between GA and ES for optimization of isothermal cavities, while in Lorenzini et al. (2014b) it was evaluated the effect of convective transfer in the cavity surfaces. Moreover, new recommendations for the best geometries are proposed for several ratios of $\mathrm{H} / \mathrm{L}$ (ratio between height and length of solid domain) solely with the employment of GA. In both studies it was noted that the number of simulations required with GA are strongly reduced in comparison with that needed with ES.

The present work has the purpose to evaluate the combined use of combinatorial optimization algorithm Simulated Annealing (SA) together with Constructal Design to evaluate the Y-shaped cavity geometry. The physical problem investigated here is the same previously studied in Lorenzini et al. (2011) and Lorenzini et al. (2014a). In this sense, results emerged by application of SA is compared with those found in literature with GA and SA.

\section{MATHEMATICAL MODEL}

Figure 1 shows the computational domain of the studied problem. The Y-shaped cavity acts removing the heat generated by solid domain. The seek for the optimal geometry of the cavity is given by variation of the degrees of freedom: $H / L, t_{1} / t_{0}, L_{l} / L_{0}$ and $\alpha$. The Finite Element Method (FEM) is used to determine the temperature field inside the solid domain and the maximum temperature is collected. The best thermal performance of the system is obtained when the maximum temperature inside the solid domain is minimized.

The solid domain, gray region in Fig. 1, has a constant conductivity $k$. Moreover, the solid generates heat at a constant volumetric rate given by $q^{\prime \prime \prime}\left(\mathrm{W} \cdot \mathrm{m}^{-3}\right)$. The outer surfaces of the solid are adiabatic, i.e., perfectly insulated. The generated heat 
current $\left(q^{\prime \prime \prime} \cdot A\right)$ is removed by the Y-shaped wall, which is maintained at a minimal temperature $\left(T_{\min }\right)$. The minimal temperature in the cavity represents a large heat transfer coefficient in such way that convective resistance can be neglected in comparison with the solid conduction resistance. This hypothesis can be associated with a large class of examples where augmentation and compactness are required, such as in the cooling packages of small-scale electronics (Lorenzini et al. 2000; Xie et al.,2010b).

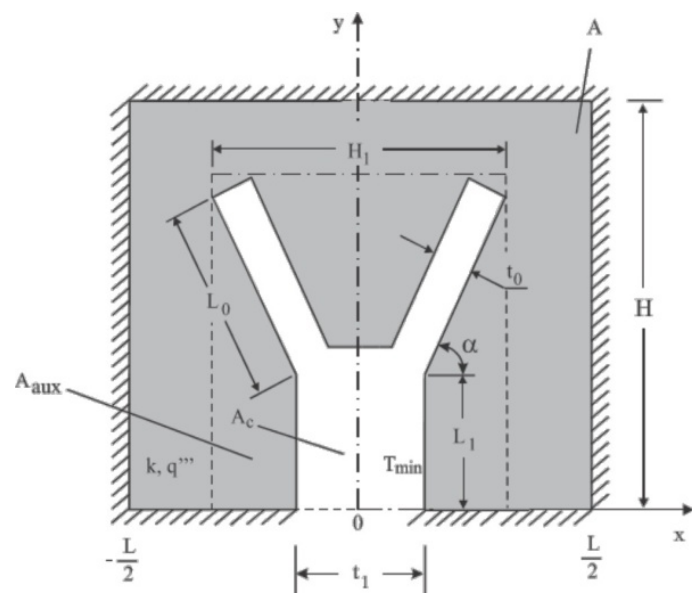

Figure 1. Computational Domain of Y-shaped cavity into a solid with internal heat generation.

The main goal here is find the optimal geometric form $\left(H / L, t_{1} / t_{0}, L_{l} / L_{0}, \alpha\right)$ which minimizes the maximum excess of temperature $\left(\theta_{\max }-\right.$ $\left.\theta_{\text {min }}\right) /\left(q^{\prime \prime \prime} A\right)$. According to Constructal Design method, this optimization must respect three constraints, total area of the solid, cavity area and auxiliary area, which are given respectively by:

$$
\begin{gathered}
A=H L \\
A_{c}=L_{1} t_{1}+2 L_{0} t_{0}+t_{1} t_{0} \cos \alpha-t_{0}^{2} \sin \alpha \cos \alpha \\
A_{\text {aux }}=\left(L_{1}+L_{0} \sin \alpha+t_{0} \cos \alpha\right)\left(2 L_{0} \cos \alpha+t_{1}\right)
\end{gathered}
$$

where the third and four terms of Eq. (2) represents the trapezoidal area that unify the simple and bifurcated branches of the Y-shaped cavity. In Eq. (3), the second and first terms between parentheses on right side of the equation represents the height and the width occupied by the cavity. The Eqs. (2) - (3) can be expressed as the cavity and auxiliary fractions in relation to total area. They are given by:

$$
\begin{array}{r}
\varphi=A_{c} / A \\
\psi=A_{\text {aux }} / A
\end{array}
$$

It is worthy to mention that the Eq. (2) is valid only for following interval: $0<\alpha<\pi / 2 \mathrm{rad}$.
The analysis that delivers the global thermal resistance as a function of the geometry consists of solving numerically the heat conduction equation along the solid region.

$$
\frac{\partial^{2} \theta}{\partial \tilde{x}^{2}}+\frac{\partial^{2} \theta}{\partial \tilde{y}^{2}}+1=0
$$

In the dimensionless process of the heat diffusion equation, the term $q$,"' $/ k$ is evidenced in all terms, given rise to the unity in the third term of Eq. (6).

The dimensionless variables can be written by:

$$
\begin{gathered}
\theta=\frac{\theta_{\max }-\theta_{\min }}{q^{\prime \prime} \frac{A}{k}} \\
\tilde{x}, \tilde{y}, \tilde{t}_{0}, \tilde{t}_{1}, \tilde{L}_{0}, \tilde{L}, \tilde{H}, \tilde{L}=\frac{x, y, t_{0}, t_{1} L_{0}, L_{1}, H, L}{A^{1 / 2}}
\end{gathered}
$$

As previously mentioned the outer surfaces of the solid are thermally insulated (adiabatic) while the cavity surfaces are maintained at minimal temperature $\theta_{\min }$. For the sake of brevity, the equations which represents the boundary conditions will not be addressed in this text. More details can be seen in the study of Biserni et al.(2004).

The dimensionless form of the Eqs. (1) - (3) are given, respectively, by:

$$
\begin{gathered}
1=\tilde{H} \tilde{L} \\
\phi=\tilde{L}_{1} \tilde{t}_{1}+2 \tilde{L}_{0} \tilde{t}_{0}+\tilde{t}_{1} \tilde{t}_{0} \cos \alpha-\tilde{t}_{0}^{2} \sin \alpha \cos \alpha \\
\psi=\left(\tilde{L}_{1}+\tilde{L}_{0} \sin \alpha+\tilde{t}_{0} \cos \alpha\right)\left(2 \tilde{L}_{0} \cos \alpha+\tilde{t}_{1}\right)
\end{gathered}
$$

The objective is minimizes the maximum excess of temperature $\theta_{\max }$ in the solid domain, given by dimensionless equation:

$$
\theta_{\max }=\frac{T_{\max }-T_{\min }}{q^{\prime \prime \prime} A / k}
$$

\section{NUMERICAL MODEL}

The function represented by Eq. (12) is determined numerically by solving Eq. (6) for the temperature field in every assumed configurations $\left(H / L, t_{1} / t_{0}, L_{l} / L_{0}, \alpha\right)$ and calculating $\theta_{\max }$ to see whether $\theta_{\max }$ can be minimized by varying the configuration. The numerical solution is performed with the Finite Element Method (FEM) (Reddy and Gartling, 1994), based on linear triangular elements, developed in the MATLAB environment, precisely the PDE (partialdifferential-equations) toolbox (MATLAB, 200). The 
grid was non-uniform in both $x$ and $y$ directions, and varied from one geometry to the next. The appropriate mesh size was determined by successive refinements (h-adaptively), increasing the number of elements four times from the current mesh size to the next mesh size, until the following criterion is satisfied:

$$
\left|\left(\tilde{\theta}_{\max }^{i}-\tilde{\theta}_{\max }^{i+1}\right) / \tilde{\theta}_{\max }^{i}\right|<1 \times 10^{-4}
$$

where $\theta^{i}$ represents the maximum value of the temperature evaluated with current mesh, $\theta^{i+1}$ represents the temperature evaluated with next refined mesh (which has four elements more than the previous mesh).

Table 1 illustrates the refinement mesh process with the aim to find a independent mesh, that does not influence final results of the temperature field. The following results were performed by using a range between 2240 and 143360 triangular elements. The independent mesh is achieved for the third refinement (35840 elements). The grid independence test is performed for a fixed geometry with the following parameters: $\phi=0.05, \psi=0.5, H / L=1.0$, $t_{1} / t_{0}=2.0, L_{l} / L_{0}=0.5, \alpha=0.94$.

Table 1. Grid independence test.

\begin{tabular}{ccc}
\hline N. Elements & $\boldsymbol{\theta}_{\max }$ & $\left|\left(\boldsymbol{\theta}_{\max }^{\mathbf{i}} \boldsymbol{\theta}^{\mathbf{i}+\mathbf{1}}{ }_{\max }\right) / \boldsymbol{\theta}_{\max }^{\mathbf{i}}\right|$ \\
\hline 2240 & 0.079046 & $5.6600 \times 10^{-4}$ \\
\hline 8960 & 0.079001 & $1.8978 \times 10^{-4}$ \\
\hline $35840 *$ & 0.078986 & $5.6971 \times 10^{-5}$ \\
\hline 143360 & 0.078982 & ------------ \\
\hline
\end{tabular}

* Independent mesh

The results obtained in grid independence test were compared to previous results found in the studies of Lorenzini et al. (2011) and Lorenzini et al. (2014a). The comparison is depicted in Tab. 2.

Table 2. Comparison between the results achieved with the present model and those showed in literature.

\begin{tabular}{cc}
\hline Reference & $\boldsymbol{\theta}_{\max }$ \\
\hline Present Work & 0.0762 \\
\hline Lorenzini et. al. (2014a) & 0.0762 \\
\hline Lorenzini et. al. (2011) & 0.0762 \\
\hline
\end{tabular}

\section{USING SIMULATED ANNEALING TO GEOMETRIC OPTIMIZATION}

The combinatorial optimization algorithm Simulated Annealing (SA) was proposed firstly in the study of Kirkpatrick et al. (1983) and it has a heuristic based in production of resistant materials, like glass or metal, submitted to annealing process. Initially the material is heated at high level temperatures and then cooled slowly to obtain the minimization of energy between the atoms of the material. The algorithm of Kirkpatrick et al. (1983) apply the optimization proposed by Metropolis et al. (1953) for various temperature levels.

Based on the heuristic presented, the algorithm SA starts the seek for the possible solutions in the search space and accept news solutions according with a probability given by Eq. (14), which depends on current iteration temperature of algorithm. The function which decreases the temperature at each iteration of the SA is named cooling schedule. For high algorithm temperatures of the annealing process the algorithm proposes distant new geometric neighbor point and has more probability to accept bad solutions, i.e., distant from the optimal shape. As the algorithm temperature decreases the new proposed neighbor points are near from the current point and the probability to accept bad solutions decreases considerably.

$$
P=\frac{1}{1+\exp \left(\frac{\Delta}{\max (T)}\right)}
$$

In Equation (14) $\Delta$ is the difference between the value returned by Objective Function of the new configuration in relation to Objective Function value for the best evaluated point. More precisely, when a new geometry neighbor is proposed in comparison with the current solution, the value of Objective Function, which it is intended to be minimized, is calculated for this new geometry (neighbor). Afterwards, it is calculated $\Delta$ subtracting the value of Objective Function for the neighbor solution and Objective Function for the current solution. The acceptance probability of solution also depends on the actual algorithm iteration. Once the numerical model used here led to an achievement of different temperatures for each degree of freedom, for calculation with Eq. (14) it is used the highest temperature of domain (MATLAB, 2000).

To apply the SA algorithm in the geometric optimization of the Y-shaped cavity, it was needed the same discretization of the space search realized in the study of Lorenzini et al. (2014a). The implementation of SA was run by MATLAB environment. More precisely, it is used the Global Optimization Toolbox with a specific function to SA execution named simulannealbnd. The SA solver of the MATLAB is extremely adaptable, i.e., it is possible to develop new algorithms for several functions as cooling schedule, annealing function or the acceptance function.

The present work applied the SA algorithm for 
the same problem previously studied in Estrada et al. (2012) and Lorenzini et al. (2014a) where the Exaustive Search (ES) and Genetic Algorithm (GA) were employed to seek for the optimal shapes. In these works all degrees of freedom $\left(H / L, t_{l} / t_{0}, L_{l} / L_{0}, \alpha\right)$ were evaluated. In the present work, the main intention was to evaluate the possibility to employ SA combined with Constructal Design for geometric optimization of complex cavity shapes. In this sense, the degree of freedom $H / L$ will be kept fixed $(H / L=$ 1.0) and the optimal shapes are obtained for various fixed values of $t_{1} / t_{0}$, finding the two times minimized maximal excess of temperature, $\left(\theta_{\max }\right)_{m m}$ and the its corresponding optimal shapes: $\left(L_{1} / L_{0}\right)_{o}$ and $\alpha_{o o}$.

The search space formed by degrees of freedom (DOF) $L_{1} / L_{0}$ and $\alpha$ was discretized in the same solution space employed for GA algorithm used in Ref. [12]. The SA was evaluated for 15 different values of DOF $L_{1} / L_{0}$, as can be seen in the matrix represented in Eq. (15), while the DOF $\alpha$ is varied in the interval $1.00 \leq \alpha \leq 1.57$ with a step of $\Delta \alpha=0.01$.

$$
L_{1} / L_{0}=\left|\begin{array}{lllll}
0.001 & 0.002 & 0.003 & 0.005 & 0.007 \\
0.01 & 0.02 & 0.03 & 0.05 & 0.07 \\
0.1 & 0.3 & 0.5 & 0.7 & 1
\end{array}\right|(15)
$$

In order to apply the SA algorithm in an appropriate search space, it is needed to develop an annealing function, i.e., the function which proposes neighbor solutions from the current point during the optimization process. The proposal of the new neighbor for the possible values of $L_{1} / L_{0}$ must respect the interval depicted in Eq. (15), and the new points for $\alpha$ can not extrapolate the interval described above with a precision of $\Delta \alpha=0.01$. A new cooling schedule (the way as the control temperature for SA algorithm in each iteration will be minimized) is proposed here and is named hybrid function. This function consists in a mixture between two functions available in MATLAB Optimization Toolbox: Exponential and Boltz. The SA algorithm used here has three available cooling functions: Exponential (default model), Fast and Boltz. These functions are presented by the following equations:

$$
\begin{gathered}
T_{i}=T_{0} \times 0.95^{k_{s a}} \\
T_{i}=\frac{T_{0}}{k_{s a}} \\
T_{i}=\frac{T_{0}}{\ln \left(k_{s a}\right)}
\end{gathered}
$$

where $k_{s a}$ is the annealing parameter, which is the same value for iteration number before reannealing, i.e., the number of iteration before the recalculation of temperature. By default, it is taking into account a value of $k_{s a}=100 . T_{i}$ represents the temperature in the current iteration and $T_{0}$ represents the initial temperature of the algorithm, that is assumed $T_{0}=$ 100 by default. In this work the proposed cooling temperature, named Boltzexp, is a hybrid form between the models described in Eq. (16) and Eq. (18). Basically, the function consists on the evaluation of the temperature obtained with the two equations and the employment of the lowest temperature obtained with both methods in the current iteration. Figs. 2 - 3 illustrate a comparative between the cooling models, including the hybrid model proposed in the present study.

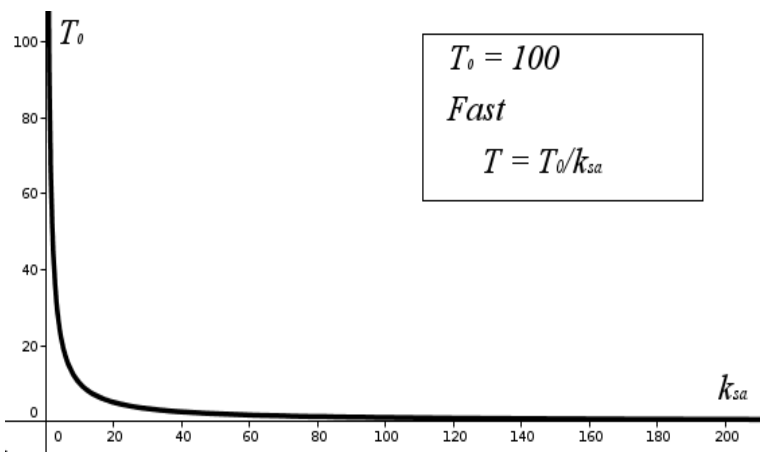

Figure 2. The behavior of algorithm temperature as a function of the number of iterations for the Fast cooling schedule of SA.

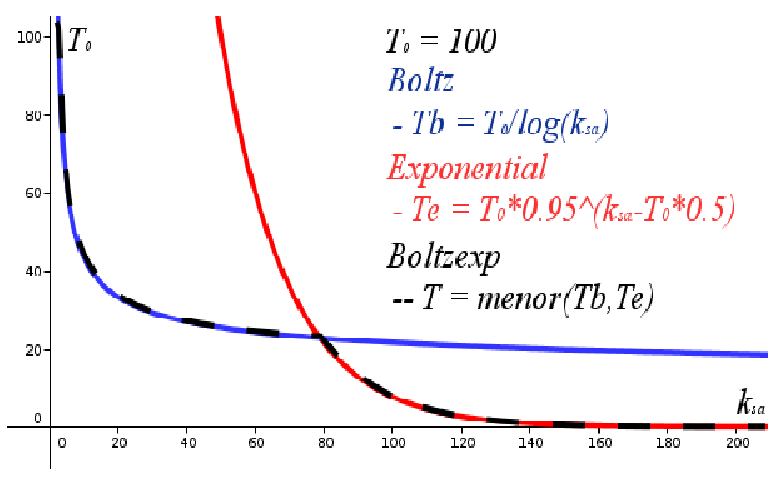

Figure 3. The behavior of temperature as a function of the number of iterations for three different cooling schedules of SA (boltz, exponential and hybrid).

It was performed sixty executions of SA algorithm for the four different cooling schedules. The execution of SA for each kind of cooling schedule led to distinct performances, which will be analyzed in next section. The algorithm stops when the maximal excess of temperature is $\theta_{\max }=0.6119$, which is the same criterion employed in the work of Lorenzini et al. (2014a) for stop of GA. The maximum number of 150 iterations without changes in the current optimal shape was also used as stop criterion for execution of SA. In spite of the establishment of this last criterion, all SA executions found the same optimal value predicted in the research of Lorenzini et al. (2014a) for the case with $H / L=1.0$ and $t_{l} / t_{0}=11.0$. 


\section{RESULTS AND DISCUSSION}

Table 3 demonstrates a comparison between the SA executions for the four kinds of cooling schedules for algorithm temperature. It is important to mention that the results of Table 3 were based in sixty executions of SA algorithm for each cooling schedule (Fast, Boltz, Exponential and Boltzexp). Once the SA algorithm is probabilistic and it has functions for generation of random numbers, it is noticed that the number of iterations (required simulations to find the optimal values) is not constant. In this sense, it is also presented in Table 3 the mean required number of iterations for achievement of optimal shapes, as well as, the lowest and highest number of simulations required for achievement of the optimal shape. It is also shown the number of times where the algorithm reaches to the optimal shape.

Table 3. Comparative between SA cooling models for temperature algorithms.

\begin{tabular}{ccccc}
$\begin{array}{c}\text { Cooling } \\
\text { Schedule (SA) }\end{array}$ & $\%$ & $\begin{array}{c}\text { Mean } \\
\text { Iter. }\end{array}$ & $\begin{array}{c}\text { Min. } \\
\text { Iter }\end{array}$ & $\begin{array}{c}\text { Max. } \\
\text { Iter. }\end{array}$ \\
\hline Fast & 50 & 129.28 & 4 & 187 \\
\hline Boltz & 72 & 206.63 & 6 & 422 \\
\hline Exp & 80 & 100.90 & 18 & 248 \\
\hline $\begin{array}{c}\text { Boltzexp } \\
\text { (hybrid) }\end{array}$ & 90 & 154.58 & 49 & 381 \\
\hline
\end{tabular}

Firstly, Table 3 shows that all models reached to the same global optimal shape. The fourth column of Table 3 shows that on the one hand the model Boltzexp is the most reliable model for achievement of the optimal geometry since it reached 54 times (of 60 executions) to the global minimal maximum excess of temperature for the $\mathrm{Y}$-shaped cavity, i.e., 90 $\%$ of executions. The other 6 executions led to local minimal temperatures and local optimal shapes. On the other hand, the Fast model reached the optimal shape only $50 \%$ of times executed, being the lowest reliability model among the studied functions. Concerning the number of iterations, it can be seen that the algorithm can reach optimal geometries (even a local optimal shapes) with few iterations (simulations), e.g., the Fast model reached to the minimal shape with only 4 iterations, while the model Boltzexp required a minimal of 49 iterations to find one optimal shape. The mean number of iterations to find the optimal shapes among the 60 executions performed for all cooling schedules are presented in sixth column of Tab. 3. The best model in this aspect is the Exp, which required approximately 100 iterations to find the optimal shape. In spite of the fact that Exp model required a number of simulations nearly $35 \%$ lower than that required for the Boltzexp model, this last model will be employed in the continuity of the work due to its elevated reliability, which is the most important aspect in the seek for the optimal shapes.

Table 4 presents a comparison between the results reached with Boltzexp cooling schedule in SA algorithm and those reached with ES (Lorenzini et al., 2011) and GA (Lorenzini et al., 2014a). The optimized geometry was performed for the following constant values of constraints and degrees of freedom: $\phi=0.05, \psi=0.3, H / L=1.0$ and $t_{1} / t_{0}=$ 11.0. The mode of number of iterations for achievement of the optimal results with SA are used for comparison with the results of literature, i.e., the number of iterations with more occurrence.

Table 4. Comparative between obtained results with ES, GA and SA for the following fixed parameters.

\begin{tabular}{ccccc}
\hline $\begin{array}{c}\text { Optimization } \\
\text { Method }\end{array}$ & $\left(\boldsymbol{L}_{\boldsymbol{l}} / \boldsymbol{L}_{\boldsymbol{0}}\right)_{\mathbf{0}}$ & $(\boldsymbol{\alpha})_{\mathrm{oo}}$ & $\left(\boldsymbol{\theta}_{\mathrm{max}}\right)_{\mathrm{mm}}$ & Iter. \\
\hline $\begin{array}{c}\text { Exhaustive } \\
\text { Search }\end{array}$ & 0,007 & 1,55 & 0,611 & 331 \\
\hline $\begin{array}{c}\text { Genetic } \\
\text { Algorithm }\end{array}$ & 0,007 & 1,55 & 0,611 & 169 \\
\hline $\begin{array}{c}\text { Simulated } \\
\text { Annealing }\end{array}$ & 0,007 & 1,55 & 0,611 & $143^{*}$ \\
\hline
\end{tabular}

* Mode of the number of iterations in $60 \mathrm{SA}$ executions with hybrid cooling model

The results of Table 4 shows that the twice minimized maximum excess of temperature, $\left(\theta_{\max }\right)_{\mathrm{mm}}$, was the same for all search mechanism. Furthermore, all methods employed found the same optimal geometries: $\left(L_{1} / L_{0}\right)_{\mathrm{o}}$ and $(\alpha)_{\mathrm{oo}}$. Concerning the number of simulations (iterations) to optimize the problem it can be observed that the search mechanisms heuristic and probabilistic (GA and SA) require a number of simulations strongly inferior to that needed with ES, allowing future studies of more complex geometries with larger number of degrees of freedom. For this specific problem, the number of simulations required by ES to find the optimal is 1.96 and 2.31 times higher than that required for GA and $\mathrm{SA}$, respectively. In spite of the lower requisition on the number of simulations with SA for this specific case, the comparison between GA and SA are not conclusive and more simulations and the study in other geometries are needed.

Figure 4 illustrates a comparison between the temperature field obtained for the optimal geometry and the extreme geometries in the valid solution space for the case where $H / L=1.0$ and $t_{1} / t_{0}=11.0$ and for the following constraints: $\phi=0.05$ and $\psi=$ 0.3 . The results presented here are obtained only with the used of SA. Figure 4(a) shows the temperature field for the lower extreme of the search space $\left(L_{1} / L_{0}\right.$ $=0.001)$, while in Fig. 4(b) it is presented the twice optimized geometry and Fig. 4(c) depicts the upper extreme of the ratio $L_{1} / L_{0}$ studied $\left(L_{l} / L_{0}=1.0\right)$. Similarly to what was noticed in previous works of Biserni et al. (2004) and Lorenzini et al. (2014a), the 
employment of Constructal Design allowed the improvement of thermal performance by means of geometrical evaluation. For the showed cases, the optimal geometry, Fig. 4(b), has a thermal performance nearly $81 \%$ and $39 \%$ superior than that achieved with the lowest and highest extremes of the ratio $L_{1} / L_{0}$, Fig. $4($ a) and $4($ c) , respectively. It is also seen that the maximum thermal performance is obtained for the geometry which led to the most homogeneous temperature field, i.e., according to the Constructal principle of "optimal distribution of imperfections” (Bejan, 1996; Bejan, 200; Bejan and Lorente, 2008).
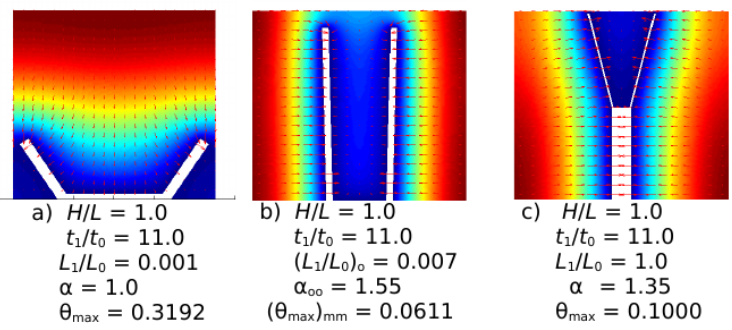

Figure 4. Temperature Fields for the geometries with various ratios of the $L_{1} / L_{0}$ : a) $L_{1} / L_{0}=0.001$, b) $L_{1} / L_{0}$ $=0.007$ (optimal), c) $L_{1} / L_{0}=1.0$.

Table 5 demonstrates the results achieved with the Simulated Annealing (SA) for different values of $t_{1} / t_{0}$ for a fixed value of $H / L=1.0$, as well as, the results reached with GA and ES in studies of Lorenzini et al. (2014a). The results showed that the search algorithm meta-heuristics and combinatorial found the same optimal shapes obtained with ES, showing that the use of Simulated Annealing (SA) algorithm is adequate for optimization of heat transfer problems combined with Constructal Design. Concerning the number of required iterations to find the optimal geometries, both methods (GA and SA) led to a strong reduction of computational effort required to obtain the optimal shapes. With exception of the case $t_{1} / t_{0}=11.0$, the GA required lower simulations than SA for every ratios of $t_{1} / t_{0}$ studied. It is worthy to mention that the number of simulations required with SA to obtain the optimal geometries has a large variation for each value of $t_{1} / t_{0}$ investigated. For instance, for $t_{1} / t_{0}$ the number of iterations varied from a minimum of 49 simulations to a maximum of 381 simulations. A similar behavior is noticed for the simulations with GA. Therefore, in spite of the advantage of GA for the results of Table 5 , the comparison between SA and GA are not conclusive. Future studies are required to evaluate the reliability of both methods (number of times where the global optimal is achieved) and the number of simulations required for problems with more degrees of freedom.

In order to evaluate the capability of SA to represent the effect of geometry over the thermal performance, Fig. 5 shows the effect of the ratio $t_{1} / t_{0}$ over the twice minimized maximal excess of temperature, $\left(\theta_{\max }\right)_{\mathrm{mm}}$, and the respective optimal shapes: $\left(L_{1} / L_{0}\right)_{\mathrm{o}}$ and $\alpha_{\mathrm{oo}}$. The results not only showed that SA is suitable to find the minimal temperature and optimal shapes, but is also suitable for prediction of the effect of the degrees of freedom (DOF) over the performance of flux system, which is extremely important in the studies of geometrical evaluation with constructal design.

Table 5. Compare between the results obtained with SA, AG and ES for a optimization with following fixes parameters: $H / L=1.0, \phi=0.05$ e $\psi=0.3$.

\begin{tabular}{|c|c|c|c|c|}
\hline \multicolumn{2}{|c|}{ Parameter / Study } & \multirow{2}{*}{$\begin{array}{c}\text { SA } \\
\\
141 \\
1.48 \\
0.001 \\
0.0700\end{array}$} & \multirow{2}{*}{$\begin{array}{c}\begin{array}{c}\text { Lorenzin } \\
\text { i et al. } \\
\text { (2014a) } \\
\text { GA }\end{array} \\
31 \\
1.48 \\
0.001 \\
0.0700\end{array}$} & \multirow{2}{*}{$\begin{array}{c}\begin{array}{c}\text { Lorenzini } \\
\text { et al. } \\
(\mathbf{2 0 1 1}) \\
\text { ES }\end{array} \\
527 \\
1.48 \\
0.001 \\
0.0700\end{array}$} \\
\hline$t_{1} / t_{0}=7.0$ & $\begin{array}{c}\text { Iter. } \\
(\alpha)_{\mathrm{oo}} \\
\left(L_{l} / L_{0}\right)_{\mathrm{o}} \\
\left(\theta_{\max }\right)_{\mathrm{m}}\end{array}$ & & & \\
\hline$t_{1} / t_{0}=10.0$ & $\begin{array}{c}\text { Iter. } \\
(\alpha)_{\mathrm{oo}} \\
\left(L_{1} / L_{0}\right)_{\mathrm{o}} \\
\left(\theta_{\max }\right)_{\mathrm{m}}\end{array}$ & $\begin{array}{c}149 \\
1.54 \\
0.001 \\
0.0639\end{array}$ & $\begin{array}{c}47 \\
1.54 \\
0.001 \\
0.0639\end{array}$ & $\begin{array}{c}649 \\
1.53 \\
0.001 \\
0.0639\end{array}$ \\
\hline$t_{1} / t_{0}=11.0$ & $\begin{array}{c}\text { Iter. } \\
(\alpha)_{\mathrm{oo}} \\
\left(L_{l} / L_{0}\right)_{\mathrm{o}} \\
\left(\theta_{\max }\right)_{\mathrm{m}}\end{array}$ & $\begin{array}{c}143 \\
1.55 \\
0.007 \\
0.0611\end{array}$ & $\begin{array}{c}169 \\
1.55 \\
0.007 \\
0.0611\end{array}$ & $\begin{array}{c}331 \\
1.55 \\
0.007 \\
0.0611\end{array}$ \\
\hline$t_{1} / t_{0}=12.0$ & $\begin{array}{c}\text { Iter. } \\
(\alpha)_{\mathrm{oo}} \\
\left(L_{1} / L_{0}\right)_{\mathrm{o}} \\
\left(\theta_{\max }\right)_{\mathrm{m}}\end{array}$ & $\begin{array}{c}119 \\
1.53 \\
0.05 \\
0.0642\end{array}$ & $\begin{array}{c}52 \\
1.53 \\
0.05 \\
0.0642\end{array}$ & $\begin{array}{c}385 \\
1.53 \\
0.05 \\
0.0642\end{array}$ \\
\hline
\end{tabular}

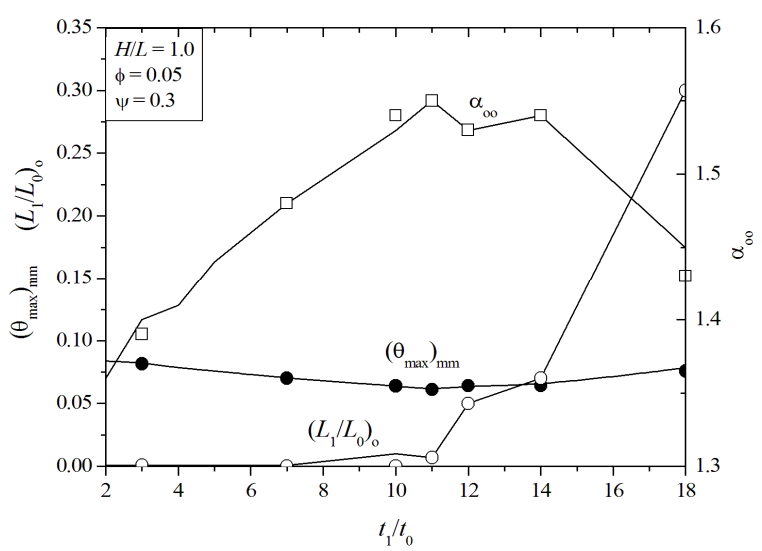

Figure 5. Comparison between results of the

Exhaustive Search Method and optimal points found with Simulated Annealing in function of $t_{1} / t_{0}$.

\section{CONCLUSIONS}

In the present work it was performed a numerical study about the employment of combined Constructal Design and Simulated Annealing (SA) for geometric evaluation and optimization of an isothermal Y-shaped cavity intruded into solid 
conducting wall with internal heat generation. The optimization results found with SA were compared with those achieved with Exhaustive Search (ES) and Genetic Algorithm (GA) presented in Biserni et al. (2004) and Lorenzini et al. (2014a).

The results obtained in the present work showed that the employment of Simulated Annealing is a suitable methodology in combination with Constructal Design for geometric optimization of heat transfer problems. Firstly, it was investigated four different cooling schedules. The hybrid method named Boltzexp increased significantly the reliability in the seek for the optimal shapes. For instance, the method Boltzexp reached to the global optimal geometry in $90 \%$ of executions performed with the code, while the method Fast achieved only in $50 \%$ of executions. In this sense, the Boltzexp function was employed in the search for the evaluation of three degrees of freedom in the Y-shaped cavity.

Concerning the seek for the optimal geometries, the twice minimized maximal excess of temperature, $\left(\theta_{\max }\right)_{\mathrm{mm}}$ and their respective optimal shapes $\left(\left(L_{1} / L_{0}\right)_{\mathrm{o}}\right.$ and $\left.\alpha_{\mathrm{oo}}\right)$ obtained with SA are the same previously predicted with ES and GA. The computational effort required with SA and GA were strongly lower than that necessary with ES. The results showed that SA combined with Constructal Design is not only suitable to find the best geometries of the Y-shaped cavities but also for prediction of the effect of geometric parameters (degrees of freedom) over the thermal performance of heat transfer problems.

Future studies will be performed to evaluate new complex geometries combining SA and GA with Constructal Design to find the optimal geometries and evaluate the effect of geometric parameters over cavities intruded into conducting solid walls.

\section{ACKNOWLEDGEMENTS}

Professor E. D. dos Santos is sponsored by FAPERGS (Process: 12/1418-4). Prof. L. A. O. Rocha thanks CNPq by finantial support.

\section{REFERENCES}

Bejan, A., 1996, Constructal-Theory Network of Conducting Paths for Cooling a Heat Generating Volume, International Journal Heat Mass Transfer, Vol. 40, No. 4, pp. 799-816.

Bejan, A., 2000, Shape and Structure, from Engineering to Nature, Cambridge University Press, Cambridge, UK.

Bejan, A., 2003, Optimal Internal Structure of Volumes Cooled by Single-Phase Forced and Natural Convection, Journal of Electronic Packaging, Vol. 125 , pp. 200-207.

Bejan, A., and Lorente, S., 2008, Design with Constructal Theory, Wiley, Hoboken.

Biserni, C., Rocha, L. A. O., and Bejan, A., 2004, Inverted Fins: Geometric Optimization of the
Intrusion into a Conducting Wall, International Journal Heat Mass Transfer, Vol. 47, pp. 2577-2586.

Eglese, R. W., 1990, Simulated Annealing: A tool for Operational Research, European Journal of Operational Research, pp. 271-281.

Estrada, E., Dos Santos, E. D., Isoldi, L. A., and Rocha, L. A. O., 2012, Genetic Algorithm and Constructal Design for Geometric Optimization of Y Shaped, in: $V$ MCSUL, $V$ Conferência Sul de Modelagem Computacional, Rio Grande - RS, Vol. 1., pp. 45-50.

Hajmohammadi, M. R., Poozesh, S., Campo, A., and Nourazar, S. S., 2013, Valuable Reconsideration in the Constructal Design of Cavities, Energy Convers Manage, Vol. 66, pp. 3340.

Kirkpatrick, S., Gelatt, C. D., and Vecchi, M. P., 1983, Optimization by Simulated Annealing, Science, New Series, Vol. 220, No. 4598, pp. 671-680.

Lorenzini, G., Biserni, C., Isoldi, L. A., Dos Santos, E. D., and Rocha, L. A. O., 2011, Constructal Design Applied to the Geometric Optimization of YShaped Cavities Embedded in a Conducting Medium, Journal Electronic Packaging, Vol. 133, pp. 041008-1 - 041008-8

Lorenzini, G., Biserni, C., Link, F. B., Isoldi, L. A., Dos Santos, E. D., and Rocha, L. A. O., 2013, Constructal Design of T-Shaped Cavity for Several Convective Fluxes Imposed at the Cavity Surfaces, Journal of Engineering Thermophysics, Vol. 22, pp. 309-321.

Lorenzini, G., Biserni, C., Estrada, E. da S. D., Dos Santos, E. D., Isoldi, L. A, and Rocha, L. A. O., 2014a, Genetic Algorithm Applied to Geometric Optimization of Isothermal Y-Shaped Cavities, Journal of Electronic Packaging, Vol. 136, pp. 031011-031020.

Lorenzini, G., Biserni, C., Estrada, E. D., Isoldi, L. A., Dos Santos, E. D., and Rocha, L. A. O., 2014b, Constructal Design of Convective Y-Shaped Cavities by Means of Genetic Algorithm, Journal of Heat Transfer, Vol. 136, pp. 071702-1-071702-10.

Lorenzini, G., Garcia, F. L., Dos Santos, E. D., Biserni, C., and Rocha, L. A. O., 2012, Constructal Design Applied to the Optimization of Complex Geometries: T-Y-Shaped Cavities with Two Additional Lateral Intrusions Cooled by Convection, International Journal of Heat and Mass Transfer, Vol. 55, pp. 1505-1512.

Lorenzini, G. L., and Rocha, L. A. O., 2000, Constructal Design of Y-Shaped Assembly of Fins, Vol. 49, pp. 4552-4557.

Lorenzini, G., and Rocha, L. A. O., 2009, Geometric Optimization of T-Y-Shaped Cavity According to Constructal Design, International Journal of Heat and Mass Transfer, Vol. 52, pp. 4675-4683.

Matlab, 2000, User's Guide, Version 6.0.088, Release 12, The Mathworks Inc., Natick, MA. 
Metropolis, N., Rosenbluth, A. W., Rosenbluth, M. N., and Teller, A. H., 1953, Equation of State Calculations by Fast Computing Machines, The Journal of Chemical Physics, Vol. 21, pp. 1088-1092.

Reddy, J. N., and Gartling, D. K., 1994, The Finite Element Method in Heat Transfer and Fluid Dynamics, CRC, Boca Raton, Florida, USA.

Xie, Z., Chen, L., and Sun, F., 2010, Geometry Optimization of T-Shaped Cavities According to Constructal Theory, Mathematical and Computer Modelling, Vol. 52, pp. 1538-1546.

Xie, Z. H., Chen, L. G., and Sun, F. R., 2010, Constructal Optimization of Twice Y-Shaped Assemblies of Fins by Taking Maximum Thermal Resistance Minimization as Objective, Science China Technological Sciences, Vol. 53, pp. 2756-2764. 